\title{
Basic Model and Profit Channel of the Integrated Energy Services
}

\author{
Shiyu Meng ${ }^{1,}$, , Jinyue Dou ${ }^{1}$, Zhuhan Long ${ }^{1}$, Ming Zeng ${ }^{1}$, Chenjun Sun ${ }^{2}$ \\ ${ }^{1}$ School of Economics and Management, North China Electric Power University, Beijing 102206, \\ China \\ ${ }^{2}$ State Grid Hebei Electric Power Company, Hebei 050000, China \\ ahbdldxmsy@126.com
}

Keywords: Energy Internet; Integrated Energy Services; Service Model; Profit Model.

\begin{abstract}
In future, with the promulgation of the energy Internet development plan and the gradual liberalization of the power sales side, the user side will appear a large variety of user service requirements and intelligent terminal access requirements. In order to realize the dynamic balance of energy supply and demand, and meet the increasingly diversified and intelligent low-carbon power demand, it is necessary to further promote the energy Internet project practice. From the current point of the view, the economic development zone, like the Tianfu new area of Sichuan province and Qianhai area of Shenzhen, is the best starting point of the energy Internet. Therefore, the paper mainly discusses the construction of energy Internet and the profitability of system operators in the area mentioned above.
\end{abstract}

\section{Basic framework of THE energy Internet}

The traditional energy planning, which is served as a basic starting point for meeting energy supply and demand, has been unable to adapt to the current economic development mode and energy structure adjustment needs. Therefore, it is a general trend to formulate the new regional energy interconnection planning based on scientific ideas. In order to save resources, realize the resources complementarity and obtain the best economic and social benefits, the paper designs the basic framework of regional energy internet.

It can be seen from the figure that the basic framework of the regional energy Internet is mainly divided into four modules, including the distributed energy supply module, system network module, demand side management module and network information processing module. Each module is connected and fused by energy flow and information flow.

In terms of the energy flow, in addition to the main existing centralized energy supply, regional energy interconnection also includes the distributed energy supply, such as distributed photovoltaic, heat pump and so on, which is connected with the main power grid by the micro grid. Both of them support the energy demand of the users in the region, and jointly drive the heating network. Both of them support the energy demand of the users in the region and drive the heating network.

In terms of the information flow, the efficient operation of the entire regional energy Internet needs to be backed by intelligent information service platform. On the one hand, through the analysis of information processing, intelligent control functions of Internet, the scheduling problem of distributed generation; on the other hand, can realize the analysis of characteristics of demand side management and users can optimize the use of electrical properties, the use of key technologies for network users. Finally, the interaction between supply and demand is achieved. On the one hand, through the information analysis and processing and the intelligent regulation and control function, the access and scheduling problem of distributed generation is solved. On the other hand, by using the key technologies of network system, the user's power consumption characteristics and energy usage characteristics are analyzed, and the demand side management and user energy optimization are realized. Finally, bilateral interaction between supply and demand is realized. 


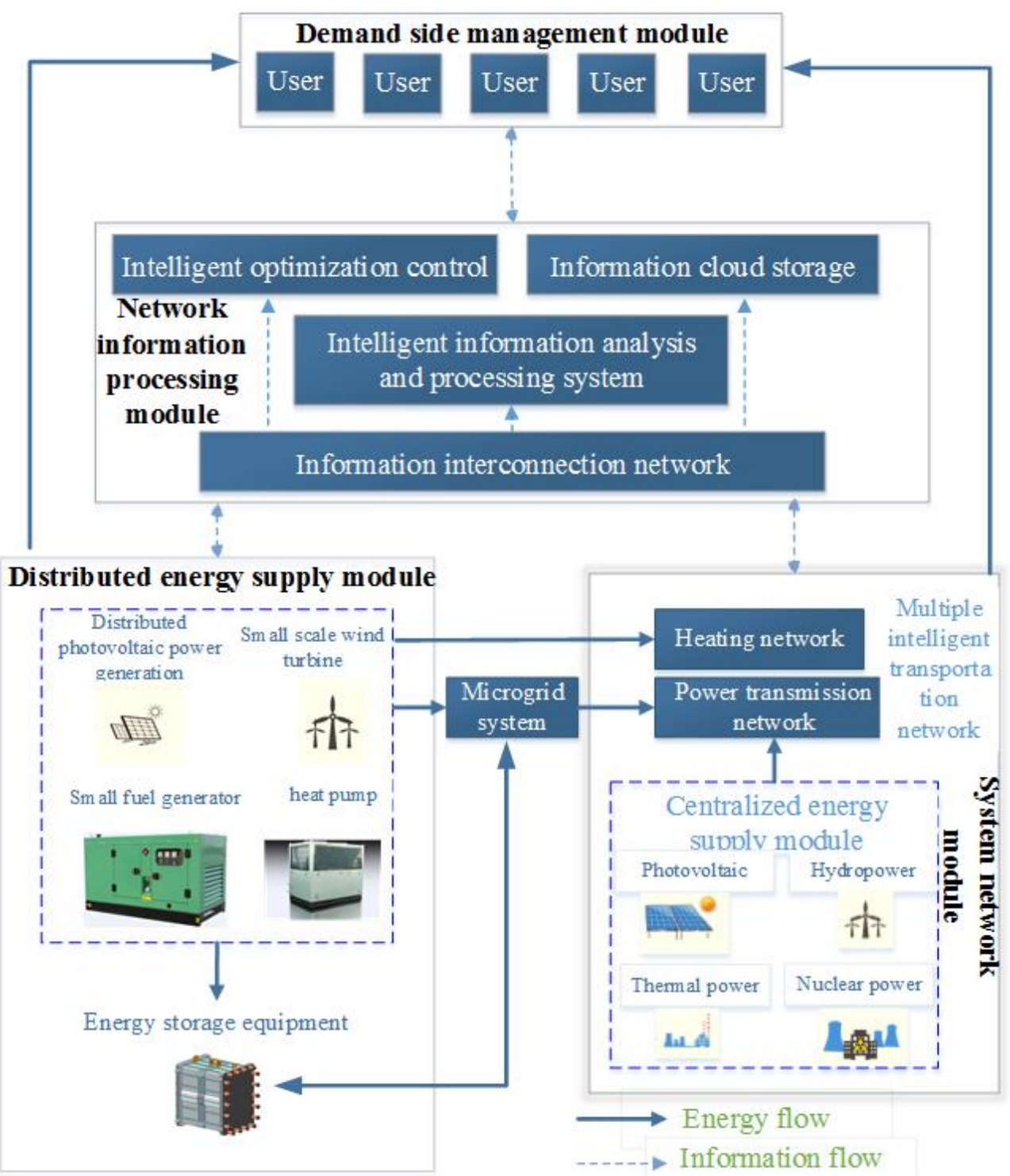

Fig 1. Basic framework of the regional energy Internet

Therefore, based on the above basic framework, system operators can provide users with integrated energy services solutions within the energy Internet range and take advantage of this. The section focuses on the analysis of the basic service model, profit model and financing model of the integrated energy services solutions.

\section{Basic service model of THE integrated energy services}

In the operation of the energy Internet, the system operators need to provide users with a variety of integrated energy services, including the power services, heating service, information service and demand side management services.

In terms of the power services, the energy of the energy Internet mainly comes from distributed clean energy, which is connected to the users through micro networks, and also connected to the large power grid through the micro grid. In the process of the system construction, according to the actual characteristics of each region, the construction of these distributed power stations should be made by means of investment by investors, user contribution or proportional contribution, and corresponding energy storage equipment shall be equipped.

In terms of the heating services, there are three channels in the heating service of the energy Internet, including the various heat pump technology, electrical heating and heating services from the public heating passages. 
In terms of the information services, system operators should provide users with energy information services, allowing users to query their own energy usage information anywhere and anytime, which can be used to compare the energy usage situation among multiple users. Therefore, data mining and analysis capabilities of the system operators is very important, which also means that operators need to build appropriate monitoring equipment and cloud analysis processing equipment at the client side and the energy supply side, and analyze user's behavior characteristics of energy usage, providing users with accurate energy supply, power demand side management, low-carbon energy-saving and other more abundant energy use program to lay the foundation.

In terms of the demand side management services, demand side management services of energy Internet are closely related to information services. Specifically, by collecting and combing the user's electricity data, system operators summarize up the user's peak and valley power consumption, power generation and other information in a certain period of time, and use technology tools to analyze the user's electricity consumption, the structure of electricity consumption and other information, providing users with an optimized energy utilization scheme at the end of the day, which will allow users to use more distributed cleaning power while reducing their own power costs.

\section{Profit model of THE integrated energy services}

For the systems operators, the biggest concern is how to profit from the energy services. The section analyzes the main profitable channels for the energy Internet,ingcluding the electricity revenue, the heating revenue, the information services revenue, and the negative watts revenue.

In terms of the electricity revenue, system operators carry out the construction of distributed power stations, and provide users with power supply in the region, which should collect electricity charges to users. Considering the investment mode of the distributed power station is different, there are mainly three kinds of profit making methods. Including full investment of the system operator, full investment in distributed generation of the users and proportional investment. The electricity charge charged by the system operators to the users is calculated as:

Distributed generation costs $=$ total investment for distributed generation $*$ equal and separated payment capital recovery factor/annual electricity consumptions + reasonable profits.

In addition, system operators also need to build micro network among distributed energy and large power grid and distributed energy users, the part costs should be included in the electricity revenue, in accordance with the similar distribution price calculation:

Microgrid costs $=[$ (power grid upgrade costs + new network investment costs $) *$ equal and separated payment capital recovery factor + system operation costs] / annual electricity consumptions + reasonable profits. When the distributed generation has an excess of power, system operators can buy this part of the power from the users and sell it to the power grid to earn the difference.

To sum up, the system operators' electricity income includes three parts, the distributed generation tariff, the microgrid costs and the distributed residual electricity price difference:

Electricity income $=$ distributed generation tariff + microgrid costs + distributed margin electricity price difference.

In terms of the heating revenue, the system operators should build distributed heating pumps for users, or actively explore other distributed heating modes. The formula is:

Heating revenue $=\Sigma$ (total heat pump investment per user * total heat pump investment per user / annual heating days + reasonable profits). The part of the information service revenue is based on user defined system, in which charges are charged in accordance with the user's customized energy information service. In terms of the negative watts revenue, "negative watts" gains are closely related to the income from information services. Which can make the peak load transfer to the base, so the system operators can sell the part of "negative watts" to the power grid companies in order to make a profit

In summary, the system operators' earnings of the energy Internet are calculated as follows: 
Total revenue $=$ electricity revenue + heating revenue + information services revenue + negative watts revenue.

\section{Financing model of THE integrated energy services}

With the gradual advancement of the energy Internet, system operators are constantly looking for their own profit model. Therefore, the paper mainly discusses the construction of energy Internet and the profitability of system operators in the area from four aspects, including the basic framework, service model and profit model, in order to achieve the best economic and social benefits

Due to broad coverage and greater investment of the construction business of the energy Internet, when the total investment is undertaken by the system operators independently, the payback period of the project is longer. As a result, it is advisable to make a contribution to take the proportional investment with the user.

There is a way to make full use of the Internet thinking and attract investors. The production costs of the currently popular TV shows are very high but it can smoothly operate and profit, the reason of which is that these TV programs have a large number of audience. Therefore, a large number of advertisers prefer to invest these programs in the form of advertising. On the one hand, the program operators gain the investment and can make a profit. On the other hand, the advertisers promote their products, which is a typical "win-win" initiative. At present, the mode of attracting foreign investment has been adopted by many network broadcast platforms and e-commerce platforms.

In terms of the energy Internet, the model mentioned above is still feasible. The system operators have a large number of users, so they can attract other investors to invest in the energy Internet construction by advertising for other investors. However, the model has a premise that there is a large number of users in the region of the energy Internet, and the audience of the advertisement must be extensive, so as to bring good implementation effect. From the current point of the view, such a mode for attracting foreign investment is more appropriate in the development zone like Tianfu new area of Sichuan province.

\section{Conclusion}

With the gradual advancement of the energy Internet, system operators are constantly looking for their own profit model. Therefore, the paper mainly discusses the construction of energy Internet and the profitability of system operators in the area from four aspects, including the basic framework, service model and profit model, in order to achieve the best economic and social benefits.

\section{Acknowledgments}

The paper is supported by the Science and Technology Program of SGCC (Implementation plan and support system of constructing power service platform by SGCC under the new policy environment).

\section{References}

[1]. Jincheng Yang, Jianbo Luo and Lihui Kang. Comprehensive Energy Service under Regional Energy Internet Structure. Shanghai Energy Conservation. 2017(03).

[2]. Hongjie Jia, Dan Wang and Xiandong Xu. Research on Some Key Problems Related to Integrated Energy Systems. Automation of Electric Power Systems. 2015(07).

[3]. Bingsen Li and Fan Wu. Development Status and Trend Research of Energy Internet. Intelligent Computer and Applications. 2017(07).

[4]. Bo Li. Discussion on the World Outlook and Methodology of Transformation and Upgrading of Power Grid Enterprises_-To Build a Comprehensive Energy Solutions and Service Platform for Enterprises. Guizhou Electric Power Technology. 2017(20). 
[5]. Xiaodan $\mathrm{Yu}, \mathrm{Xiandong} \mathrm{Xu}$ and Shuoyi Chen. A Brief Review to Integrated Energy System and Energy Internet. Transactions of China Electrotechnical Society. 2016(31).

[6]. Chaoyang Dong, Junhua Zhao and Fuquan Wen. From Smart Grid to Energy Internet: Basic Concept and Research Framework. Automation of Electric Power Systems. 2014(15).

[7]. Hongbin Sun, Qinglai Guo and Zhaoguang Pan. Energy Internet: Concept, Architecture and Frontier Outlook. Automation of Electric Power Systems. 2015(19). 